Anaesthesist 2020 - 69:825

https://doi.org/10.1007/s00101-020-00849-9

Online publiziert: 24. September 2020

(c) Springer Medizin Verlag $\mathrm{GmbH}$, ein Teil von Springer Nature 2020

Die Empfehlungen der Sepsisleitlinien fassen die derzeitigen Erkenntnisse zur Therapie zusammen. Wie in allen gängigen Leitlinien werden Standards erhoben, die aber nicht allen Patienten gerecht werden können, da die individuelle Immunantwort und damit auch das Ansprechen auf eine Therapie interindividuell variieren. Damit kann Unsicherheit entstehen, ob die Standardtherapie tatsächlich zu einer signifikanten Verbesserung der Erkrankung beiträgt.

\section{Das ist neu!}

Seymour et al. haben anhand der $\mathrm{Pa}$ tientendaten aus 3 Observationsstudien $(n=63.858)$ und 3 randomisierten, kontrollierten Sepsisstudien $(n=4737)$ neue Sepsisphänotypen ( $\alpha, \beta, \gamma$ und $\delta$ ) klassifiziert, die sich im Hinblick auf Laborresultate, Notwendigkeit einer Intensivtherapie, Organversagen und Letalität unterschieden [1].

So neigten Patienten mit einem $\delta$-Phänotyp häufiger zu hepatischer und kardiovaskulärer Dysfunktion, während $\beta$-Phänotypen wahrscheinlicher ein Nierenversagen hatten. Variiert man den Anteil der Phänotypen in den untersuchten Studienpopulationen (aus der PROWESS-[2], ACCESS-[3] und ProCESS-Studie [4]), können die Studien unterschiedliche Ergebnisse zeigen. So ist die Wahrscheinlichkeit eines Vorteils der „Early-goal-directed“-Therapie (EGDT) bei 35\%, wenn mehrheitlich Patienten mit a-Phänotyp in der ProCESS-Studienpopulation sind. Finden sich dagegen in der gleichen Studienpo-

\title{
T. Schürholz
}

Klinik f. Anästhesiologie und Intensivtherapie, Universitätsmedizin Rostock, Rostock, Deutschland

\section{Was ist neu ... Standardisierung vs. Individualisierung in der Intensivmedizin. (K)ein Widerspruch?}

pulation wenigstens $50 \%$ Patienten mit einem $\delta$-Phänotyp, besteht eine $60 \%$ ige Chance, einen Nachteil durch EGDT zu belegen. In diesen Simulationen waren die Schlussfolgerungen über den Nutzen oder Schaden einer Therapie abhängig von der Verteilung der Sepsisphänotypen. Da die Phänotypen anhand von klinisch routinemäßig verfügbaren $\mathrm{Da}$ ten unterschieden werden, könnten also eine Abschätzung der Prognose und auch eine Individualisierung der Therapie ab dem Aufnahmezeitpunkt möglich sein [1]. Obwohl diese retrospektive Untersuchung Limitationen hat, zeigt sie doch, dass eine sinnvolle Standardtherapie wie EGDT unterschiedlich effektiv bei individuellen Patienten sein kann. Dass die Standardtherapiestrategien wie Volumengabe und Vasopressortherapie in der Sepsis individualisiert zu einem besseren Ergebnis führen können, zeigte auch eine Untersuchung zur Unterstützung bei Therapieentscheidungen durch künstliche Intelligenz („,artificial intelligence“ AI) [5]. In dieser Simulation führte die AI-gestützte Sepsistherapie in optimierter Dosierung zu einem besseren Überleben. Ein Überschreiten der empfohlenen Volumentherapie und ein Abweichen bei der empfohlenen Vasopressordosierung resultierte in höherer Letalität [5].

\section{Fazit für die Praxis}

Die Standardtherapie der Sepsis beschreibt die grundlegenden Ansätze, die bei den Erkrankten angewendet werden sollen. Dass diese Standard- therapie in Dosis, Zeitpunkt und evtl. auch der Dauer individuell an den $\mathrm{Pa}$ tienten angepasst sein muss, belegen die Studien von Seymour et al. [1] und Komorowski et al. [5]. Standardisierung und Individualisierung sind also kein Widerspruch, sondern ergänzen sich in der an den Patienten angepassten Optimierung der Sepsistherapie.

\section{Korrespondenzadresse}

Univ.-Prof. Dr. T. Schürholz

Klinik f. Anästhesiologie und Intensivtherapie, Universitätsmedizin Rostock

Schillingallee 35, 18057 Rostock, Deutschland tobias.schuerholz@med.uni-rostock.de

Interessenkonflikt. T. Schürholz: B. Braun Melsungen, Fresenius AG, Adrenomed AG.

\section{Literatur}

1. Seymour CW, Kennedy JN, Wang S et al (2019) Derivation, validation, and potential treatment implications of novel clinical phenotypes for sepsis. JAMA 321:2003-2017

2. Bernard GR, Vincent JL, Laterre PF et al (2001) Efficacy and safety of recombinant human activated protein C for severe sepsis. N Engl J Med 344:699-709

3. Opal SM, Laterre PF, Francois B et al (2013) Effect of eritoran, an antagonist of MD2-TLR4, on mortality in patients with severe sepsis: the ACCESS randomized trial. JAMA 309:1154-1162

4. Pro Cl, Yealy DM, Kellum JA et al (2014) A randomized trial of protocol-based care for early septic shock. NEngl J Med 370:1683-1693

5. Komorowski M, Celi LA, Badawi O et al (2018) The artificial intelligence clinician learns optimal treatment strategies for sepsis in intensive care. Nat Med 24:1716-1720 